\title{
Women and children last? Shaking up exclusion criteria for vaccine trials
}

\author{
Is it time to shift to a model of 'inclusion unless otherwise justified' in trials of vaccines for \\ pandemics?
}

$\mathrm{T}$ he COVID-19 pandemic has turned many things on its head, including routine approaches to the development of vaccines. The urgency of the pandemic has meant that the lengthy timelines of clinical trials, stepwise adaptations and eventual programmatic rollouts via primary-care systems have been truncated to achieve rapid development. Unprecedented commitments from science, industry, regulators and funders have driven this fast-tracked progress.

However, an area in which there has been a willful lack of progress has been the inclusion of children and pregnant women in late-stage vaccine clinical trials for SARS-CoV-2.

To achieve a rapid route to licensure, while addressing commercial concerns centered on legal and financial liabilities, SARS-CoV-2 vaccine trials have generally excluded pregnant women, who have a higher likelihood of adverse events. This has little to do with any vaccine issues but everything to do with pregnancy itself. Miscarriages, premature births and stillbirths are unfortunate but not infrequent outcomes of pregnancies. It is important to emphasize that so far, no vaccines routinely recommended in pregnancy (vaccines against tetanus, influenza and whooping cough) have shown an increase in rates of poor pregnancy outcomes in vaccinated pregnant women versus those who are not vaccinated. However, for the inclusion of pregnant women in trials of novel vaccines, including those directed against SARS-CoV-2, tighter observations during pregnancy and after delivery are needed in order to confirm that any adverse outcomes of pregnancy are not related to vaccination. Follow-up of the newborns is also required, given that theoretically, a vaccine given in pregnancy could also affect the offspring.

Due to these added complexities, manufacturers shy away from including pregnant people in vaccine studies, and therefore, no safety data currently exist for pregnant women or infants.

But the frontline healthcare workforce responding to SARS-CoV-2 is disproportionately made up of people who can get pregnant, and a substantial portion of the workforce could be pregnant as the rollout of SARS-CoV-2 vaccines commences. Because of the lack of safety data, they could not currently be offered any SARS-CoV-2 vaccine, which would deprive them of access to this preventive and potentially life-saving measure.

And what about children? Clinical trials of new vaccines specifically aimed at vaccinating children normally proceed via a so-called 'age de-escalation' strategy: in early-phase trials, the vaccine is usually given to healthy adults first, then to sequentially younger children to make sure that it is equally safe and immunogenic. If it is, phase 3 trials can then proceed in the final target group, which is often infants.

In the context of COVID-19, however, young children are not seen as a prime target group for vaccination, as they have largely remained asymptomatic.

By an abundance of caution or lack of commercial interest, thus far, younger children have not been included in the SARS-CoV-2 clinical trials, and therefore there are also no safety data for this group, similar to the situation for pregnant women. Planned SARS-CoV-2 vaccination campaigns currently exclude young children, but this might represent a barrier to achieving the levels of community immunity needed to bring this pandemic under control.

A shift to a paradigm of 'inclusion unless justified otherwise' is overdue. Pregnant women and parents need to be asked for their views and opinions about getting involved in trials and rollouts, both for themselves and for their young children.

Right now, funding for safety and immunogenicity studies of both groups should be made available as soon as efficacious vaccines have received regulatory approval.

Going forward, trials need to be sufficiently funded to set up appropriate safety follow-ups and registries of participants to determine outcomes for pregnancies and infants.
This is not a 'blind' choice. Given the experience with vaccines against tetanus, influenza, whooping cough and, lately, Ebola, in pregnancy, there are already familiar types of licensed vaccines and adjuvants for other infectious diseases with established safety databases. A strong case can therefore be made for the inclusion of young children and pregnant women during late-stage trials of SARS-CoV-2 vaccines through the use of similar platforms in order to gather important safety and immunogenicity data in these populations. This would facilitate the early inclusion of young children and pregnant women in the vaccine rollout after regulatory approval.

To assist standardization of the interpretation of pregnancy outcomes during clinical trials, agreed definitions, such as those developed by the Global Alignment of Immunization Safety Assessment project, already exist. They have in particular been tested during ongoing trials of vaccines specifically developed to be given to pregnant women in order to prevent infections in the newborn, such as vaccines against respiratory syncytial virus and group B streptococcus.

The knowledge gained by the scientific community and vaccine manufacturers in implementing trials of these novel vaccines, including the establishment of routine procedures for recruitment, monitoring and pregnancy registries, can now also help in moving forward safely to include rather than automatically exclude young children and pregnant women in trials for SARS-CoV-2 vaccines from the moment late-stage trial protocols are designed. This would allow the rollout of SARS-CoV- 2 vaccines to move ahead with the inclusion of these important groups without considerable delay.

\section{Beate Kampmann (D)}

The Vaccine Centre, London School of Hygiene \& Tropical Medicine, London, UK.

$\bigotimes_{\text {e-mail: beate.kampmann@lshtm.ac.uk }}$

Published online: 13 January 2021 https://doi.org/10.1038/s41591-020-01199-0

Competing interests

The author declares no competing interests. 